\title{
Cyst fluid hemoglobin species in endometriosis and its malignant transformation: The role of metallobiology
}

\author{
TAKUYA IWABUCHI $^{1}$, CHIHARU YOSHIMOTO $^{2}$, HIROSHI SHIGETOMI $^{2}$ and HIROSHI KOBAYASHI ${ }^{2}$ \\ ${ }^{1}$ Department of Research and Development, Metallogenics Co., Ltd., Chiba 260-0856; \\ ${ }^{2}$ Department of Obstetrics and Gynecology, Nara Medical University, Kashihara, Nara 634-8522, Japan
}

Received February 21, 2015; Accepted March 4, 2016

DOI: $10.3892 / \mathrm{ol} .2016 .4383$

\begin{abstract}
The aim of the present study was to assess the relative concentrations of hemoglobin $(\mathrm{Hb})$ species in endometriosis as a possible indicator of malignancy. Electronic absorption spectroscopy was employed to quantify the $\mathrm{Hb}$ species present in the cyst fluid collected from 8 patients with endometriosis-associated ovarian cancer (EAOC), and compared with those present in the cyst fluid of 35 patients with benign endometriotic cysts. The $620 / 580 \mathrm{~nm}$ ratio in the electronic absorption spectrum, which was used as a surrogate indicator of the methemoglobin (metHb)/(oxyhemoglobin+metHb) ratio, was measured in each cyst fluid by ultraviolet/visible grating spectrophotometric microplate reader. The optimal cutoff value was defined according to the analysis of receiver operating characteristic (ROC) curve. The sensitivity and specificity of detection were calculated on the basis of the cutoff value to differentiate EAOC from endometriosis. The $620 / 580 \mathrm{~nm}$ ratio of cyst fluid in EAOC patients was much lower than that measured in women with benign cysts $(0.389 \pm 0.266$ vs. $0.666 \pm 0.188$, $\mathrm{P}=0.021)$. ROC curve analysis performed using 0.35 as the optimal cutoff value indicated that the $620 / 580 \mathrm{~nm}$ ratio had a sensitivity, specificity, positive predictive value (PPV) and negative predictive value of $62.5,100.0,100.0$ and $92.1 \%$, respectively, in the diagnosis of EAOC. In conclusion, metHb is one of the most abundant $\mathrm{Hb}$ species in benign cysts, and the absorption $620 / 580 \mathrm{~nm}$ ratio of cyst fluid exhibits high specificity and PPV as a surveillance test for the early detection of malignant transformation of endometriosis. Thus, metallobiology highlights diverse features involved in $\mathrm{Hb}$ homeostasis and the pathogenesis of malignant transformation of endometriosis.
\end{abstract}

Correspondence to: Professor Hiroshi Kobayashi, Department of Obstetrics and Gynecology, Nara Medical University, 840 Shijo-cho, Kashihara, Nara 634-8522, Japan

E-mail: hirokoba@naramed-u.ac.jp

Key words: endometriosis, malignant transformation, iron, oxyhemoglobin, methemoglobin

\section{Introduction}

Endometriosis is a hormone-dependent benign disease that affects $\sim 10 \%$ of women during reproductive age (1). Retrograde flow of menstrual blood may lead to endometriosis (2). Individuals with endometriosis require a long follow-up, with particular attention paid not only to dysmenorrhea and infertility but also to potential malignant transformation (3). In Japan, <1\% of women with endometriosis develop ovarian cancer (3-6). Substantial histological evidence indicates that endometriosis is associated with ovarian cancer, particularly endometrioid carcinoma and clear cell carcinoma (5). Although the pathogenesis of carcinogenesis remains unclear, it is assumed that malignant transformation may be derived from pathways associated with hyperestrogenism, chronic inflammation and oxidative stress (7). Inflammation and particularly oxidative stress, which is secondary to the influx of erythrocytes and subsequent hemolysis that occurs during retrograde menstruation and inner hemorrhage in endometriotic lesions, may be one of the causative factors in carcinogenesis (4). Hemoglobin ( $\mathrm{Hb})$, heme and iron may have a significant impact on oxidative stress in the development of endometriosis and malignant transformation (8). Therefore, metallobiology studies on $\mathrm{Hb}$-mediated oxidative damage and antioxidant response are required.

The two most common redox states exhibited by iron are ferrous $\left(\mathrm{Fe}^{2+}\right.$, reduced) and ferric $\left(\mathrm{Fe}^{3+}\right.$, oxidized) (8). In methemoglobin (metHb), the iron in the heme moiety of $\mathrm{Hb}$ is oxidized from the ferrous $\left(\mathrm{Fe}^{2+}\right)$ to the ferric state $\left(\mathrm{Fe}^{3+}\right)$, while oxyhemoglobin (oxyHb) contains iron in the ferrous state $\left(\mathrm{Fe}^{2+}\right)(8)$. In $\mathrm{Hb}$, iron is predominately in the ferrous state, and participates in redox chemistry producing free radicals (9). It has been previously reported that excess of $\mathrm{Hb}$, heme and iron may be harmful to cells and may lead to cell death (10). Furthermore, sublethal oxidative stress may be linked with carcinogenic processes rather than cell death $(11,12)$. However, no data are currently available regarding the concentrations of oxyHb and metHb present in the $\mathrm{Hb}$ species of endometriotic cyst fluid.

Therefore, the purpose of the present study was to investigate the proportion of metHb in benign cysts and endometriosis-associated ovarian cancer (EAOC), and to demonstrate the efficacy of this biomarker in the differential diagnosis of EAOC vs. benign cysts. 


\section{Materials and methods}

Study population. The present study was approved by the Ethics Committee of Nara Medical University (Kashihara, Japan). A prospective single-center cohort study was performed in accordance with the Declaration of Helsinki (13). All patients signed written consent forms to participate in the present study. Patients were recruited from the Nara Medical University Hospital (Kashihara, Japan). From December 2012 to July 2013, 43 women underwent operation, 35 of whom received a diagnosis of benign endometriotic cysts, while 8 received a diagnosis of EAOC. Cyst fluid samples were collected at the time of surgery. Specimens were immediately aliquoted and frozen at $-80^{\circ} \mathrm{C}$ within $1 \mathrm{~h}$ of collection. Clinicopathologic data obtained from patients' medical records included age at the time of surgery, tumor size, stage and pathology. None of the patients had been consuming antioxidant vitamin supplements for $\geq 2$ months.

Measurement of the electronic absorption spectra in cyst fluid. The electronic absorption spectra of the common and different species of $\mathrm{Hb}$ in cyst fluid were investigated in EAOC and benign cysts. Frozen-thawed cyst fluid was diluted with phosphate-buffered saline (Takara Bio, Inc., Otsu, Japan) and added to a 96-well spectrophotometric plate (Cosmo Bio Co., Ltd., Tokyo, Japan). The electronic absorption spectra of the samples were measured with a grating spectrophotometric microplate reader (SH-1200; Corona Corporation, Sanjo, Japan). Previous studies demonstrated that a peak at $620 \mathrm{~nm}$ in the spectrum indicates specific absorption of metHb, whereas fairly sharp peaks located at 545 and $580 \mathrm{~nm}$ represent the absorption spectrum of a mixture of oxyHb and metHb (14-17).

There was marked light scattering in the samples, as evidenced by the non-zero absorbance at $700 \mathrm{~nm}$. As a simple approach, the baseline was adopted using linear regression based on a linear function (Fig. 1). The absorption bands between wavelengths $605-655 \mathrm{~nm}$ and between wavelengths $565-605 \mathrm{~nm}$ were selected. The absorbance values at 620 and $580 \mathrm{~nm}$ were followed as surrogate markers of the relative concentrations of the $\mathrm{Hb}$ species present in the samples, and the ratio of the absorption measured at $620 \mathrm{~nm}$ divided by that measured at $580 \mathrm{~nm}$ was calculated as a predictive marker of the metHb/(oxyHb+metHb) ratio present in the cyst fluids.

Statistical analysis. Data are presented as the mean \pm standard deviation. Differences between the groups of patients were estimated by Mann-Whitney U test. Analyses were performed by SPSS version 21.0 (IBM SPSS, Armonk, NY, USA). Two-sided $\mathrm{P}<0.05$ was considered to indicate a statistically significant difference.

\section{Results}

Study population. The clinicopathological characteristics of the two groups of patients are shown in Table I. Since there were differences in age and tumor size, the clinical characteristics were not balanced. The median age in the benign cysts and EAOC groups were 40.0 years (range, 21.0-55.0 years) and 49.5 years (range, 38.0-53.0 years), respectively $(\mathrm{P}=0.012)$. The mean cyst size was $6.7 \mathrm{~cm}$ (range, $2.7-19.3 \mathrm{~cm}$ ) in case
A

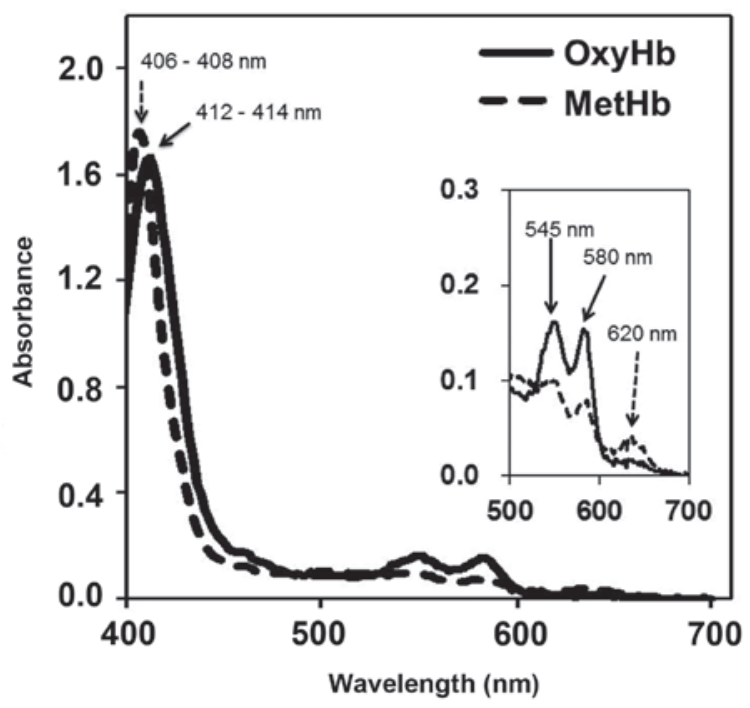

B

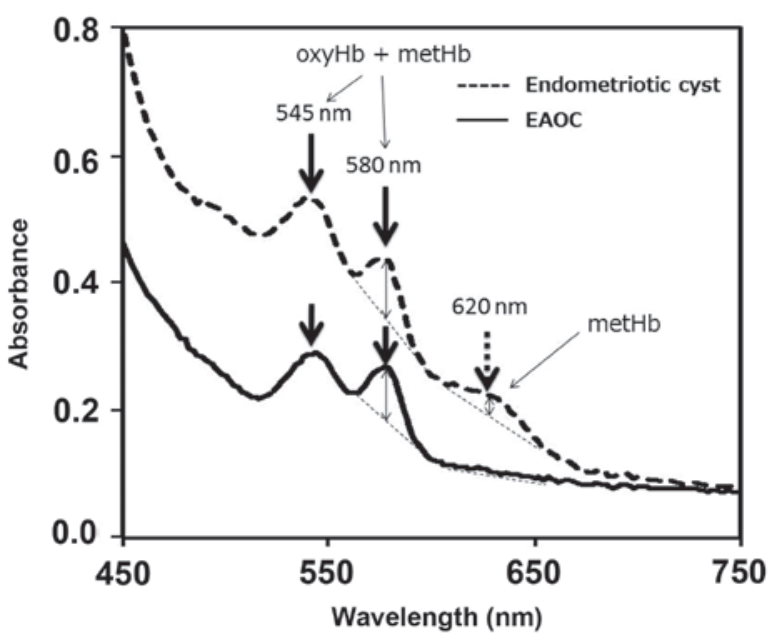

Figure 1. Electronic absorption spectra. (A) Electronic absorption spectra of purified oxyHb and metHb in phosphate-buffered saline, which were used as standards. Bold line, $30 \mathrm{mg} / \mathrm{ml}$ oxyHb; dotted line, $30 \mathrm{mg} / \mathrm{ml} \mathrm{metHb}$. The electronic absorption spectra of the different $\mathrm{Hb}$ species were represented as a function of the wavelength at the visible/near-infrared region of the electromagnetic spectrum, between 400 and $700 \mathrm{~nm}$. The insert shows the region of the higher power image on the electromagnetic spectrum between $500-700 \mathrm{~nm}$. (B) Electronic absorption spectra of the common and variable species of $\mathrm{Hb}$ present in the cyst fluid of patients with endometriotic cysts and EAOC. The spectrum shown is a representative image of the independent experiments conducted to evaluate the absorption spectra of cyst fluids. Bold line, cyst fluid sample obtained from EAOC patients; dotted line, cyst fluid sample obtained from patients with endometriotic cysts. The electronic absorption spectra of the different $\mathrm{Hb}$ species were represented as a function of the wavelength at the visible/near-infrared region of the electromagnetic spectrum, between 450 and $750 \mathrm{~nm}$. MetHb exhibits absorption peaks at $\sim 620,545$ and $580 \mathrm{~nm}$, while oxyHb exhibits absorption peaks at $\sim 545$ and $580 \mathrm{~nm}$. oxyHb, oxyhemoglobin; metHb, methemoglobin; EAOC, endometriosis-associated ovarian cancer; $\mathrm{Hb}$, hemoglobin.

of benign cysts and $10.6 \mathrm{~cm}$ (range, $4.4-14.3 \mathrm{~cm}$ ) in case of EAOC; thus, the size of the primary tumor was significantly larger in the EAOC than in the benign cyst group $(\mathrm{P}=0.003)$.

Absorption spectra of hemoglobin species in cyst fluids derived from endometriotic cysts and EAOC. As represented in Fig. 1A, purified oxyHb solution exhibited double 
Table I. Demographic and clinical characteristics of the study population.

\begin{tabular}{|c|c|c|c|}
\hline Characteristics & Endometriotic cysts & EAOC & P-value \\
\hline Number & 35 & 8 & - \\
\hline Median age (range), years & $40.0(21.0-55.0)$ & $49.5(38.0-53.0)$ & 0.012 \\
\hline Mean tumor size (range), $\mathrm{cm}$ & $6.7(2.7-19.3)$ & $10.6(4.4-14.3)$ & 0.003 \\
\hline \multicolumn{4}{|l|}{ FIGO stage } \\
\hline Ia & - & 1 & - \\
\hline $\mathrm{Ib}$ & - & 1 & - \\
\hline Ic & - & 6 & - \\
\hline \multicolumn{4}{|l|}{ Pathology } \\
\hline Endometriosis & 35 & & \\
\hline Clear cell adenocarcinoma & - & 5 & - \\
\hline Endometrioid adenocarcinoma & - & 2 & - \\
\hline Mucinous adenocarcinoma & - & 1 & - \\
\hline
\end{tabular}

EAOC endometriosis-associated ovarian cancer, FIGO, Federation of Gynecology and Obstetrics (18).

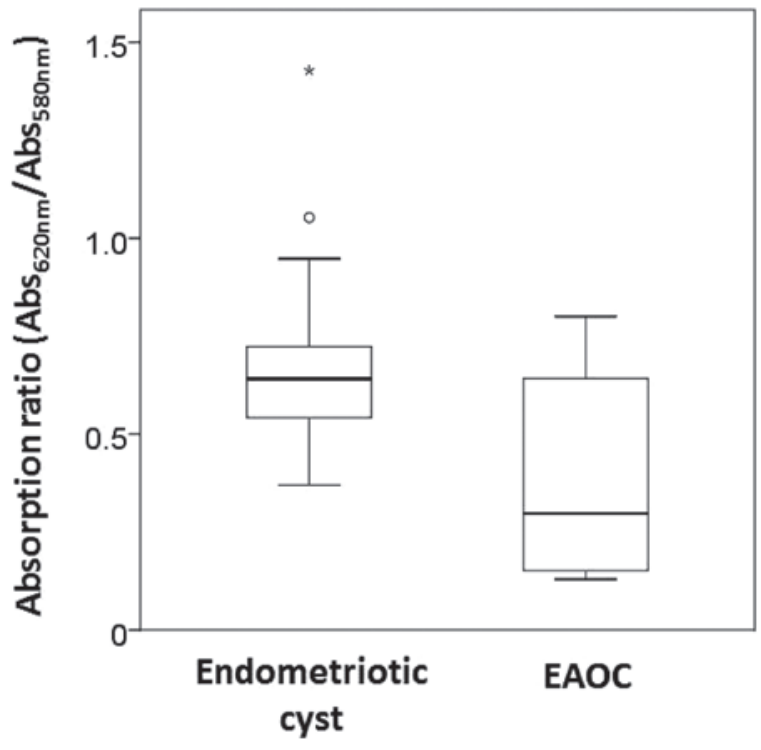

Figure 2. Comparison of the absorption ratio at 620 vs. $580 \mathrm{~nm}$ of cyst fluids derived from 35 patients with benign endometriotic cysts and 8 patients with endometriosis-associated ovarian cancer. ${ }^{*} \mathrm{P}=0.021$. EAOC, endometriosis-associated ovarian cancer; Abs, absorption.

sharp peaks at 545 and $580 \mathrm{~nm}$ in the electronic absorption spectrum, while purified metHb produced three absorption spectral peaks at 545, 580 and $620 \mathrm{~nm}$, respectively. The intense peak at $620 \mathrm{~nm}$ appears to be composed of metHb, while the peaks at 545 and $580 \mathrm{~nm}$ are probably the result of a mixture of oxyHb and metHb. Fig. $1 \mathrm{~B}$ represents the typical absorption spectra of $\mathrm{Hb}$ species in cyst fluids from endometriotic cysts and EAOC in the spectral range of 450-750 nm. The experimental data demonstrated good reproducibility, with the electronic absorption spectra exhibiting prominent peaks at $\sim 545,580$ and $620 \mathrm{~nm}$ in benign cysts, and 545 and $580 \mathrm{~nm}$ in EAOC. At 545 and $580 \mathrm{~nm}$, the absorption spectra were not obviously different between benign cysts and EAOC. Notably, the present results revealed a consistent difference

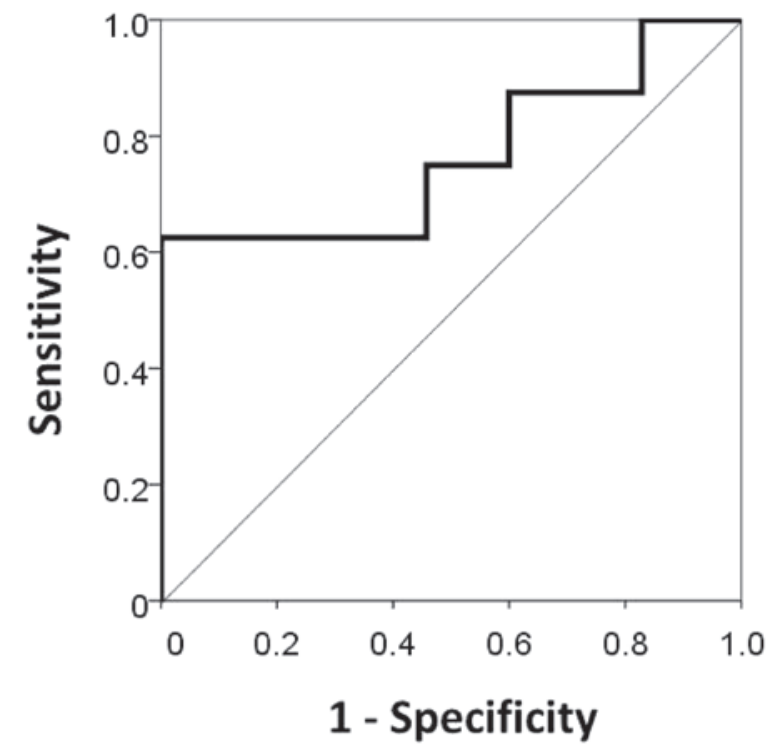

Figure 3. Receiver operating characteristic curve analysis of the absorption ratio at $620 / 580 \mathrm{~nm}$ of cyst fluid samples derived from individuals with benign endometriosis and patients with endometriosis-associated ovarian cancer.

in the region of $620 \mathrm{~nm}$ between the absorption spectrum of benign cysts and that of EAOC. These differences in the absorption spectra may have been caused by differences in the degree of metHb formation between benign cysts and EAOC.

The relative concentrations of $\mathrm{Hb}$ species in cyst fluid was assessed as a possible indicator of malignancy in endometriosis. For that purpose, quantification of the $620 / 580 \mathrm{~nm}$ ratio in the absorption spectra was used as a surrogate marker of the metHb/(oxyHb+metHb) ratio present in the sample. Fig 2 contains box and whisker plots representing the median levels and interquartile range (box) of the absorption at $620 \mathrm{~nm} /$ absorption at $580 \mathrm{~nm}$ ratio for each of the groups studied. The $620 / 580 \mathrm{~nm}$ absorption ratio was significantly 
lower in the cyst fluid of EAOC individuals than in that of patients with benign cysts $(0.389 \pm 0.266$ vs. $0.666 \pm 0.188$, $\mathrm{P}=0.021)$.

Receiver operating characteristic (ROC) curve analysis for prediction of optimal cutoff values. The results of ROC curve analysis based on the detection of 8 EAOC patients and 35 women with benign endometriosis are shown in Fig. 3. The optimal cutoff value was determined by analyzing the ROC curve among EAOC and endometriosis. When the cutoff value was set to 0.35 , the sensitivity, specificity, positive predictive value and negative predictive value of the $620 / 580 \mathrm{~nm}$ absorption ratio in the diagnosis of EAOC vs. benign endometriosis were $62.5,100.0,100.0$ and $92.1 \%$, respectively.

\section{Discussion}

In the present study, the absorption ratio at $620 / 580 \mathrm{~nm}$ was assessed to quantify the relative concentrations of $\mathrm{Hb}$ species present in cyst fluid collected from patients with EAOC vs. those present in cyst fluid collected from benign cysts, as a possible indicator of malignancy in endometriosis. To the best of our knowledge, the present study reports for the first time that the $620 / 580 \mathrm{~nm}$ absorption ratio was altered in cyst fluid of individuals with EAOC, compared with those with benign cysts. The relative amount of oxyHb and metHb released into the cyst fluid was spectrophotometrically measured. Patients with endometriosis exhibited higher levels of metHb in their cyst fluid, compared with EAOC patients. This indicated that the $620 / 580 \mathrm{~nm}$ ratio was a significant discriminator for malignant transformation in women with endometriosis, and the optimal cutoff point to predict malignant transformation was 0.35 .

$\mathrm{Hb}$ may be converted to metHb in the endometriotic cyst fluid solution. When erythrocytes are lysed in endometriotic cysts, $\mathrm{Hb}$, heme and iron are released from lysed red blood cells into the cyst fluid space (19). Harmful reactive oxygen species (ROS) such as $\mathrm{O}_{2}{ }^{--}$are produced during $\mathrm{Hb}$ autoxidation of oxyHb-Fe ${ }^{2+}$ to metHb-Fe ${ }^{3+}$, as follows:

$$
\mathrm{Hb}-\mathrm{Fe}^{2+}+\mathrm{O}_{2} \Leftrightarrow \mathrm{Hb}-\mathrm{Fe}^{2+}-\mathrm{O}_{2} \rightarrow \mathrm{Hb}^{-} \mathrm{Fe}^{3+}+\mathrm{O}_{2}^{-{ }^{-}}(20-22) .
$$

Furthermore, generation of free oxygen radicals $(\mathrm{OH})$ responsible for cellular destruction by the ferrous state of iron has been documented in Haber-Weiss-Fenton chemistry:

$$
\mathrm{Fe}^{2+}+\mathrm{H}_{2} \mathrm{O}_{2} \rightarrow \mathrm{Fe}^{3+}+\mathrm{OH}^{-}+\mathrm{OH}^{-}(23,24) .
$$

Therefore, in endometriotic cyst fluid, a burst of ROS (including $\mathrm{O}_{2}{ }^{--}$and $\mathrm{OH}$ ) would be generated via $\mathrm{Hb}$ autoxidation and Haber-Weiss-Fenton reaction. These data suggest that the high levels of metHb observed in patients with endometriosis may be may be due to excess free radical formation. Excess ROS may oxidize lipids, proteins and DNA, and subsequently induce apoptosis and cell death $(25,26)$.

Notably, significantly lower levels of metHb were present in the cyst fluid of EAOC patients, compared with those present in the cyst fluid of patients with benign cysts. This finding suggests the presence of potential endogenous antioxidant compounds, with a reduction of the total oxidant status. Reductase systems may catalyze the reduction of ferric iron $\mathrm{Fe}^{3+}$ to the ferrous $\mathrm{Fe}^{2+}$ state (22), suggesting a role for antioxidant response in the pathogenesis of EAOC. Antioxidant activity may be upregulated to address the redox imbalance in a subset of endometriotic cells, which may be exposed to sublethal levels of ROS and able to adapt to cell survival (27-29). The present data support the idea that excess free radicals may lead to cell damage that culminates in cell death, whereas persistent exposure to sublethal ROS with an increased antioxidant state increases the tumorigenic potential of endometriotic cells $(24,30)$.

Limitations of the present study include the lack of a significant number of patients and the generalizability of the observed results. In addition, the $\mathrm{pH}$ may affect the features of the metHb spectra (31), but the this was not evaluated in the present study. Furthermore, we hypothesize that other absorbing species are likely to be present in cyst fluid, including hemichromes, and possibly ferrylhemoglobin, sulfhemoglobin or deoxyhemoglobin (deoxyHb), although the levels of each of these species may be relatively low. Also, the absorbance of the samples at each wavelength is the result of the sum of the products of the extinction coefficients of each of the species present in the sample, multiplied by the concentration of those species (8). Therefore, the ratio of the absorption measured at $620 \mathrm{~nm}$ divided by that measured at $580 \mathrm{~nm}$ does not accurately report the metHb/(oxyHb+metHb) ratio. A more accurate method to determine the concentration of $\mathrm{Hb}$ species using absorption spectroscopy would be to collect data using an integrating sphere detector, so that scattered light is collected and true absorbance could be obtained. Thus, the present findings warrant further studies on the utilization of an integrating sphere detector. Finally, the method reported in the present study is not a serum-based test; thus, it may not exhibit the characteristics of the reliable, fast, low-cost, efficient bedside tests and non-invasive techniques that are clinically required for the assessment of the metHb/(oxyHb+metHb) ratio in cyst fluid. A number of studies have used broadband diffuse optical spectroscopy and frequency-domain near-infrared spectroscopy (NIRS) to assess tissue concentrations of metHb, oxyHb and deoxyHb $(32,33)$. Lee et al $(33)$ reported noninvasive, quantitative in vivo measurements of metHb formation using broadband diffuse optical spectroscopy (33). NIRS may provide new avenues for future determination of the metHb/(oxyHb+metHb) ratio through transvaginal application of this modality. We hypothesize that these techniques would offer the potential for bedside measurement of $\mathrm{Hb}$ and hemodynamics.

Despite the aforementioned limitations, the results of the present metallobiology study are discussed in association with the current understanding of the pathogenesis of malignant transformation of endometriosis. Thus, if the value of the $620 / 580 \mathrm{~nm}$ ratio in the absorption spectrum of cyst fluid derived from patients with endometriosis is $<0.35$, it may be considered a possible marker to accurately identify patients with endometriosis at greatest risk of malignant transformation. A reduced $620 / 580 \mathrm{~nm}$ absorption ratio may aid to identify patients who require immediate clinical evaluation for cancer surveillance and surgical attention.

In conclusion, the present study examined the diagnostic utility of the absorption ratio at $620 / 580 \mathrm{~nm}$ of cyst fluid in 
endometriosis and its malignant transformation. Further studies with larger patient groups are required to identify an optimal cutoff value of this ratio for accurate diagnosis of EAOC.

\section{Acknowledgements}

The present study was supported by a Grant-in-Aid for Scientific Research from the Ministry of Education, Science and Culture of Japan (Tokyo, Japan; grant no. 26293361). Dr Naoto Furukawa, Dr Shozo Yoshida, Dr Ryuji Kawaguchi and Dr Seiji Kanayama (Department of Obstetrics and Gynecology, Nara Medical University Hospital) were responsible for clinical management and patient registration.

\section{References}

1. Cramer DW and Missmer SA: The epidemiology of endometriosis. Ann NY Acad Sci 955: 11-22, 2002.

2. Oral E and Arici A: Pathogenesis of endometriosis. Obstet Gynecol Clin North Am 24: 219-331, 1997.

3. KobayashiH,Sumimoto K,KitanakaT,Yamada Y,SadoT,SakataM, Yoshida S, Kawaguchi R, Kanayama S, Shigetomi H, et al: Ovarian endometrioma - risks factors of ovarian cancer development. Eur J Obstet Gynecol Reprod Biol 138: 187-193, 2008.

4. Higashiura Y, Kajihara H, Shigetomi $\mathrm{H}$ and Kobayashi $\mathrm{H}$ : Identification of multiple pathways involved in the malignant transformation of endometriosis (Review). Oncol Lett 4: 3-9, 2012.

5. Tanase Y,Furukawa N, Kobayashi H and Matsumoto T: Malignant transformation from endometriosis to atypical endometriosis and finally to endometrioid adenocarcinoma within 10 years. Case Rep Oncol 6: 480-484, 2013.

6. Kobayashi H, Sumimoto K, Moniwa N, Imai M, Takakura K, Kuromaki T, Morioka E, Arisawa K and Terao T: Risk of developing ovarian cancer among women with ovarian endometrioma: A cohort study in Shizuoka, Japan. Int J Gynecol Cancer 17: 37-43, 2007.

7. Worley MJ, Welch WR, Berkowitz RS and $\mathrm{Ng} \mathrm{SW}$ : Endometriosis-associated ovarian cancer: A review of pathogenesis. Int J Mol Sci 14: 5367-5379, 2013.

8. Iwabuchi T, Yoshimoto C, Shigetomi $\mathrm{H}$ and Kobayashi $\mathrm{H}$ : Oxidative stress and antioxidant defense in endometriosis and its malignant transformation. Oxid Med Cell Longev 2015: 848595, 2015.

9. Singh AV, Vyas V, Montani E, Cartelli D, Parazzoli D, Oldani A, Zeri G, Orioli E, Gemmati D and Zamboni P: Investigation of in vitro cytotoxicity of the redox state of ionic iron in neuroblastoma cells. J Neurosci Rural Pract 3: 301-310, 2012.

10. Uttara B, Singh AV, Zamboni P and Mahajan RT: Oxidative stress and neurodegenerative diseases: A review of upstream and downstream antioxidant therapeutic options. Curr Neuropharmacol 7: 65-74, 2009.

11. Kensler TW, Egner PA, Taffe BG and Trush MA: Role of free radicals in tumor promotion and progression. Prog Clin Biol Res 298: 233-248, 1989.

12. Kamendulis LM, Jiang J, Xu Y and Klaunig JE: Induction of oxidative stress and oxidative damage in rat glial cells by acrylonitrile. Carcinogenesis 20: 1555-1560, 1999.

13. Reynolds T: Declaration of Helsinki revised. J Natl Cancer Inst 92: 1801-1803, 2000

14. Zijlstra WG, Buursma A and Meeuwsen-van der Roest WP Absorption spectra of human fetal and adult oxyhemoglobin, de-oxyhemoglobin, carboxyhemoglobin, and methemoglobin. Clin Chem 37: 1633-1638, 1991.
15. Bellavia L, DuMond JF, Perlegas A, Bruce King S and Kim-Shapiro DB: Nitroxyl accelerates the oxidation of oxyhemoglobin by nitrite. Nitric Oxide 31: 38-47, 2013.

16. Shibayama N, Sugiyama K and Park SY: Structures and oxygen affinities of crystalline human hemoglobin $C$ ( $\beta 6$ Glu- $>$ Lys $)$ in the R and R2 quaternary structures. J Biol Chem 286: 33661-33668, 2011.

17. Keszler A, Piknova B, Schechter AN and Hogg N: The reaction between nitrite and oxyhemoglobin: A mechanistic study. J Biol Chem 283: 9615-9622, 2008.

18. Prat J; FIGO Committee on Gynecologic Oncology: Staging classification for cancer of the ovary, fallopian tube, and peritoneum. Int J Gynaecol Obstet 124: 1-5, 2014.

19. Kumar S and Bandyopadhyay U: Free heme toxicity and its detoxification systems in human. Toxicol Lett 157: 175-188, 2005.

20. Umbreit J: Methemoglobin - it's not just blue: A concise review. Am J Hematol 82: 134-144, 2007.

21. Scott MD, van den Berg JJ, Repka T, Rouyer-Fessard P, Hebbel RP, Beuzard Y and Lubin BH: Effect of excess alpha-hemoglobin chains on cellular and membrane oxidation in model beta-thalassemic erythrocytes. J Clin Invest 91: 1706-1712, 1993

22. Kawano $\mathrm{T}$, Pinontoan $\mathrm{R}$, Hosoya $\mathrm{H}$ and Muto $\mathrm{S}$ : Monoamine-dependent production of reactive oxygen species catalyzed by pseudoperoxidase activity of human hemoglobin. Biosci Biotechnol Biochem 66: 1224-1232, 2002.

23. Muñoz-Bravo C, Gutiérrez-Bedmar M, Gómez-Aracena J, García-Rodríguez A and Navajas JF: Iron: Protector or risk factor for cardiovascular disease? Still controversial. Nutrients 5: 2384-2404, 2013.

24. Akatsuka S, Yamashita Y, Ohara H, Liu YT, Izumiya M, Abe K, Ochiai M, Jiang L, Nagai H, Okazaki Y, et al: Fenton reaction induced cancer in wild type rats recapitulates genomic alterations observed in human cancer. PLoS One 7: e43403, 2012.

25. Belcher JD, Beckman JD, Balla G, Balla J and Vercellotti G: Heme degradation and vascular injury. Antioxid Redox Signal 12: 233-248, 2010.

26. Kobayashi H, Yamada Y, Kanayama S, Furukawa N, Noguchi T, Haruta S, Yoshida S, Sakata M, Sado T and Oi H: The role of iron in the pathogenesis of endometriosis. Gynecol Endocrinol 25: 39-52, 2009.

27. Sova H, Kangas J, Puistola U, Santala M, Liakka A and Karihtala P: Down-regulation of 8-hydroxydeoxyguanosine and peroxiredoxin II in the pathogenesis of endometriosis-associated ovarian cancer. Anticancer Res 32: 3037-3044, 2012.

28. Balla G, Jacob HS, Balla J, Rosenberg M, Nath K, Apple F, Eaton JW and Vercellotti GM: Ferritin: A cytoprotective antioxidant strategem of endothelium. J Biol Chem 267: 18148-18153, 1992.

29. Khan MA, Sengupta J, Mittal S and Ghosh D: Genome-wide expressions in autologous eutopic and ectopic endometrium of fertile women with endometriosis. Reprod Biol Endocrinol 10: $84,2012$.

30. Fiedor J and Burda K: Potential role of carotenoids as antioxidants in human health and disease. Nutrients 6: 466-488, 2014

31. Stroes JW and van Rijn HJ: Quantitative measurement of blood pigments in cerebrospinal fluid by derivative spectrophotometry. Ann Clin Biochem 24: 189-197, 1987.

32. Fantini S, Franceschini MA, Maier JS, Walker SA, Barbieri B and Gratton E: Frequency-domain multichannel optical detector for noninvasive tissue spectroscopy and oximetry. Opt Eng 34: 32-42, 1995.

33. Lee J, El-Abaddi N, Duke A, Cerussi AE, Brenner M and Tromberg BJ: Noninvasive in vivo monitoring of methemoglobin formation and reduction with broadband diffuse optical spectroscopy. J Appl Physiol (1985) 100: 615-622, 2006. 